\title{
Gravitational-Wave Signature of a First-Order Quantum Chromodynamics Phase Transition in Core-Collapse Supernovae
}

\author{
Shuai Zha๑* and Evan P. O'Connor® \\ The Oskar Klein Centre, Department of Astronomy, Stockholm University, AlbaNova, SE-106 91 Stockholm, Sweden \\ Ming-chung Chu॰ and Lap-Ming Lin $\odot$ \\ Department of Physics and Institute of Theoretical Physics, The Chinese University of Hong Kong, \\ Shatin, N.T., Hong Kong S.A.R., China \\ Sean M. Couch $(1)$ \\ Department of Physics and Astronomy, Michigan State University, East Lansing, Michigan 48824, USA; \\ Department of Computational Mathematics, Science, and Engineering, Michigan State University, \\ East Lansing, Michigan 48824, USA; \\ Joint Institute for Nuclear Astrophysics-Center for the Evolution of the Elements, \\ Michigan State University, East Lansing, Michigan 48824, USA \\ and National Superconducting Cyclotron Laboratory, Michigan State University, \\ East Lansing, Michigian 48824, USA
}

(Received 29 May 2020; accepted 6 July 2020; published 29 July 2020)

\begin{abstract}
A first-order quantum chromodynamics (QCD) phase transition (PT) may take place in the protocompact star (PCS) produced by a core-collapse supernova (CCSN). In this work, we study the consequences of such a PT in a nonrotating CCSN with axisymmetric hydrodynamic simulations. We find that the PT leads to the collapse of the PCS and results in a loud burst of gravitational waves (GWs). The amplitude of this GW burst is $\sim 30$ times larger than the postbounce GW signal normally found for nonrotating CCSN. It shows a broad peak at high frequencies $(\sim 2500-4000 \mathrm{~Hz})$ in the spectrum, has a duration of $\lesssim 5 \mathrm{~ms}$, and carries $\sim 3$ orders of magnitude more energy than the other episodes. Also, the peak frequency of the PCS oscillation increases dramatically after the PT-induced collapse. In addition to a second neutrino burst, the GW signal, if detected by the ground-based GW detectors, is decisive evidence of the first-order QCD PT inside CCSNe and provides key information about the structure and dynamics of the PCS.
\end{abstract}

DOI: 10.1103/PhysRevLett.125.051102

Introduction.-Quarks are confined in hadrons such as protons and neutrons at low temperatures and densities. Nonetheless, free quarks should exist in the early Universe when the temperature is extremely high $\left(k_{B} T \gtrsim 150 \mathrm{MeV}\right)$ [1]. They may also exist in the cold and superdense interior of compact stars with a density above the nuclear saturation density $\left(\rho_{\text {sat }} \simeq 2.6 \times 10^{14} \mathrm{~g} \mathrm{~cm}^{-3}\right) \quad$ [see, e.g., [2-4] ]. Moreover, a first-order quantum chromodynamics (QCD) phase transition (PT), i.e., hadron-quark PT, may take place in the protocompact stars (PCS) produced by a core-collapse supernova (CCSN) [5-7] or binary neutronstar merger [8,9]. Such a PT can result in a more compact PCS and even collapse of the PCS to a black hole (BH). For

Published by the American Physical Society under the terms of the Creative Commons Attribution 4.0 International license. Further distribution of this work must maintain attribution to the author(s) and the published article's title, journal citation, and DOI. Funded by Bibsam.
CCSNe, this can provide an additional energy source for the explosion [7], and leads to interesting observational consequences, such as a second neutrino burst [5] and the production of rare $r$-process elements [10].

A galactic CCSN is a yet-undiscovered candidate gravitational-wave $(\mathrm{GW})$ source for ground-based $\mathrm{GW}$ detectors [11]. The GWs from a CCSN, in combination with the neutrino and electromagnetic signals, will boost our understanding of the CCSN explosion mechanism [12]. Sophisticated multidimensional simulations have predicted the GW signals emitted by rotational CCSNe, the oscillations of the protoneutron stars (PNS), and the standing accretion shock instability [see, e.g., [13-17]]. In the meantime, the collapse of a neutron star (NS) to a quark star has been studied in [18-20] and GW emission is also found in this scenario. However, in these studies the collapse is triggered artificially by using different equations of state (EOS) for the construction of NS and the hydrodynamic simulation. It is unclear whether the PT-induced collapse of PCS in CCSNe can leave an imprint in the GW 
signal. In this Letter, we demonstrate the effects of a first-order QCD PT on the GW signal from a nonrotating CCSN, with two-dimensional simulations and a simplified hybrid EOS including hadrons and quarks.

Methods.-Equation of state: To study a first-order QCD PT in CCSNe, we use a hybrid EOS from Refs. [5,21,22]. This EOS employs the STOS EOS [23] for the hadronic phase and the MIT bag model EOS [2] for the quark phase, with the Gibbs construction for the mixed phase [24]. The bag constant has a value of $B=(165 \mathrm{MeV})^{4}$. The Gibbs construction enforces global charge neutrality; therefore, it allows for different charge fractions for the hadronic and quark portions in the mixed phase. This leads to a smooth transition from the hadronic phase to the mixed phase (at around $\rho_{\text {sat }}$ for the composition of a CCSN core), and the pressure is continuously increasing. A pure quark phase is realized at densities higher than $\sim 3.5 \rho_{\text {sat }}$, which is similar to the values of those more sophisticated hybrid EOSs [7,9]. More information about the hybrid EOS can be found in Refs. [5,21,23].

For this hybrid EOS, there exists a stable branch of the hot third family of compact stars [4] with a pure quark core (see Fig. 2 in [25]) for matter properties similar to the CCSN core (entropy $\simeq 3 k_{B}$ /baryon and lepton number fraction $\sim 0.4)$. The maximum mass for the third family $\left(\sim 1.50 M_{\odot}\right)$ is larger than that of the second family whose core is in the mixed phase. As we will see, this unique property is important for the dynamics of the PCS.

FLASH simulation: We carry out CCSN simulations in two dimensions with the assumption of axisymmetry, using the FLASH code [26] with an "M1" scheme for the neutrino transport [27]. We take the $12-M_{\odot}$, solar-metallicity, presupernova progenitor $s 12$ from [28] as the initial conditions. To apply general-relativistic approximations, gravity is calculated with the Case A formulation of [29]. Unlike previous simulations with the FLASH code, we include the lapse function in the Euler equations to mimic the time-dilation effect in general relativity (see the modified Euler equations and some numerical tests in [30], also see [33]). This is found to affect the GW frequency significantly after the PT-induced collapse. A cylindrical grid with adaptive mesh refinement is used. It extends out to $2 \times 10^{9} \mathrm{~cm}$ in radius and $\pm 2 \times 10^{9} \mathrm{~cm}$ along the cylindrical axis, with a finest resolution of $150 \mathrm{~m}$. We extract the plus GW strain $h_{+}$from our Newtonian simulation using the standard quadrupole formula [34].

Results.-Dynamics:To show the consequences of the PT, we run two simulations with the same settings, one using the hybrid EOS and the other using the STOS EOS. The resulting dynamics are shown in the upper panel of Fig. 1. The iron core of the $s 12$ model collapses to above $\rho_{\text {sat }}$ and bounces at $t_{b} \simeq 151 \mathrm{~ms}$ for both EOSs. At $t_{b}$, the core of the hybrid EOS has already entered the mixed phase with a central quark mass fraction $X_{q}=18 \%$. However, because the hybrid EOS transitions smoothly from the pure hadronic phase to the mixed phase, the PCS remains in the mixed phase with a low $X_{q}$ shortly after $t_{b}$. The bounce shock turns into an accretion shock and stalls at $\sim 150 \mathrm{~km}$ at $t_{b}+50 \mathrm{~ms}$ and begins receding inward.

During the accretion phase, the central density $\rho_{c}$ of the PCS with the hybrid EOS is always larger than that of the PNS with the STOS EOS and $X_{q}$ continuously increases. The mass of the PCS grows and reaches the maximum of the second family for the hybrid EOS at $\sim t_{b}+286 \mathrm{~ms}$. The PCS becomes unstable against gravity and experiences a second dynamical collapse. The central density $\rho_{c}$ grows to $1.5 \times 10^{15} \mathrm{~g} \mathrm{~cm}^{-3}\left(\sim 6 \rho_{\text {sat }}\right)$ and the PCS core enters the pure quark phase $\left(X_{q}=1\right)$.

The pure quark core bounces in less than $1 \mathrm{~ms}$ at $t_{2 b}$ as the PCS enters the new stable branch of the third family and $\rho_{c}$ drops to $\sim 5 \rho_{\text {sat }}$. This bounce shock expands quickly to explode the outer envelope. At the end of the simulation, the mean shock radius extends out to $\sim 1500 \mathrm{~km}$ with an explosion energy of $\sim 2.0 \times 10^{50} \mathrm{erg}$. The PT-induced collapse is associated with a second neutrino burst with more electron antineutrinos than electron neutrinos (lower panel of Fig. 1), which is consistent with the results of the spherically symmetric simulation in [5].

Gravitational waves:In Fig. 2 we show the GW waveforms $h_{+}(t)$ up to $400 \mathrm{~ms}$ after the first bounce $\left(\sim t_{2 b}+113 \mathrm{~ms}\right)$ extracted from both simulations. We assume that the distance from the source is $10 \mathrm{kpc}$. The signal from $t_{b}$ to $\sim t_{b}+50 \mathrm{~ms}$ comes from the prompt convection behind the stalling accretion shock. It is followed by an episode of continuous emission from the oscillations of the PCSs [35]. There is no qualitative

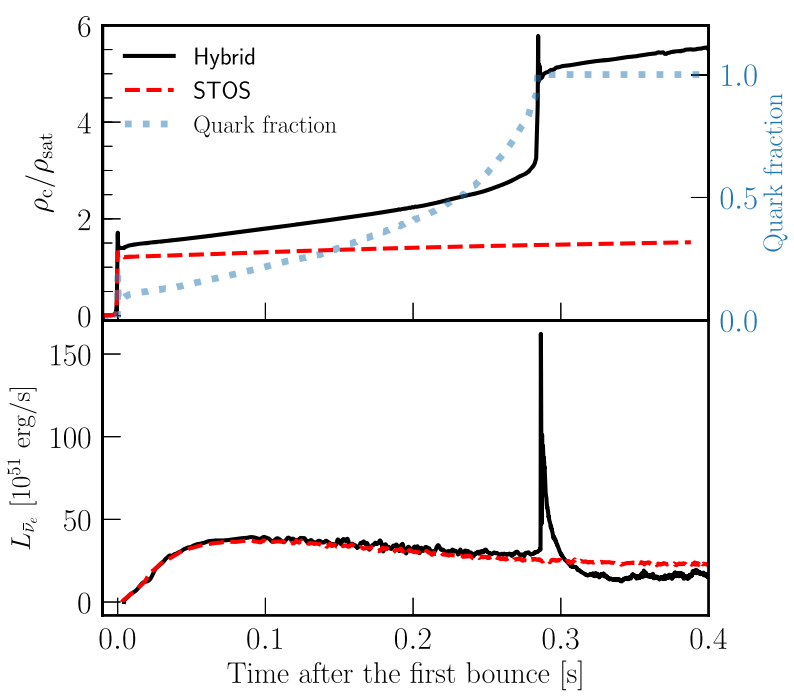

FIG. 1. Upper panel: evolution of central density $\rho_{c}$ after the first bounce for simulations using the hybrid (black solid) and STOS (red dashed) EOSs. Also shown is the quark fraction at the center of the PCS with the hybrid EOS (blue dashed). Lower panel: luminosity curves of the electron antineutrino $\left(\bar{\nu}_{e}\right)$ for the two simulations. 


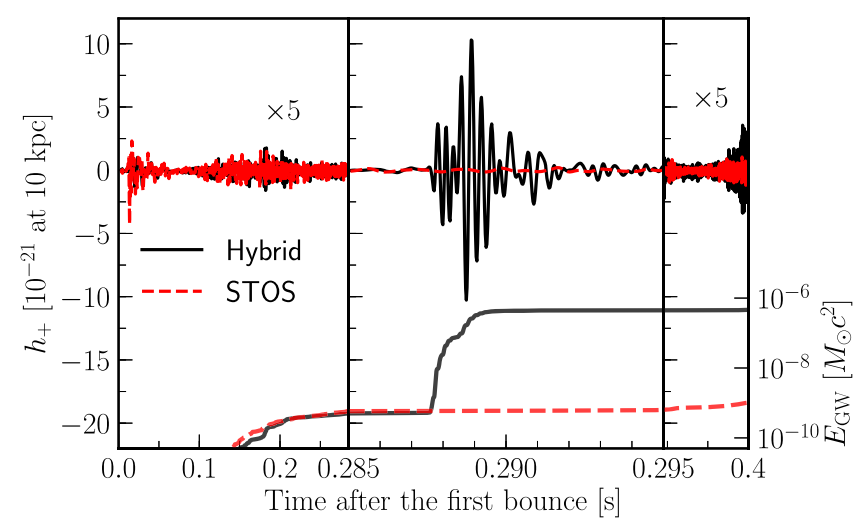

FIG. 2. GW waveforms $h_{+}(t)$ extracted from the CCSN simulations using the hybrid (black solid) and STOS (red dashed) EOSs. The $10 \mathrm{~ms}$ window around the second bounce $t_{2 b} \simeq t_{b}+$ $287 \mathrm{~ms}$ is stretched in time to clearly show the loud GW burst. $h_{+}$ in the other two episodes are multiplied by a factor of 5 to emphasize the contrast in the amplitude. The cumulative emitted GW energies are shown at the bottom.

difference between the two waveforms until $t_{2 b}$ and the cumulative emitted GW energies are quantitatively similar (bottom of Fig. 2). In accord with the more compact PCS, the peak GW frequency for the hybrid EOS is always higher than that for the STOS EOS.

Around $t_{2 b}$, the PT-induced collapse results in a burst of GW emission with a much larger amplitude than those of earlier episodes. In Fig. 2, the $10 \mathrm{~ms}$ window around $t_{2 b}$ is stretched in time to show clearly this burst, which is associated with the PT-induced collapse and bounce. The maximum amplitude of $h_{+}$reaches $10^{-20}$ and $\sim 30$ times larger than those of the other episodes. The energy carried by this burst is $\sim 4.6 \times 10^{-7} M_{\odot} c^{2}$, which is $\sim 3$ orders of magnitudes more than the GW energy of the other episodes (and also that of the signal for the STOS EOS). Our numerical test shows that this $\mathrm{GW}$ burst results from asphericities developed between $t_{b}$ and $t_{2 b}$ [30]. After this burst, the amplitude damps quickly to the same level as before $t_{2 b}$. This part of signal should come from the oscillations of the PCS with a pure quark core.

A time-dependent spectrum (or spectrogram) is useful for understanding the emission mechanisms of GWs, as well as designing efficient detection strategies. Figure 3 shows the spectrogram of the GW signal extracted from the simulation using the hybrid EOS. We use a Kaiser window with a width of $25 \mathrm{~ms}$ for the short-time Fourier transform except for around $t_{2 b}$ where a width of $10 \mathrm{~ms}$ is used. Before $t_{2 b}$, the spectral evolution is similar to that of the STOS EOS (see [30]). The GW peak frequency is continuously increasing, in accord with the evolution of the Brunt-Väisälä frequencies $f_{\mathrm{BV}}$ [Eq. (3) in [30] ] at densities between $10^{11}$ and $10^{12} \mathrm{~g} \mathrm{~cm}^{-3}$ (blue band in Fig. 3), which is approximately the PCS surface [36]. Around $t_{2 b}$, the GW burst has a much higher frequency $(\sim 2500-4000 \mathrm{~Hz})$. This is related to the change of the dominant GW emission

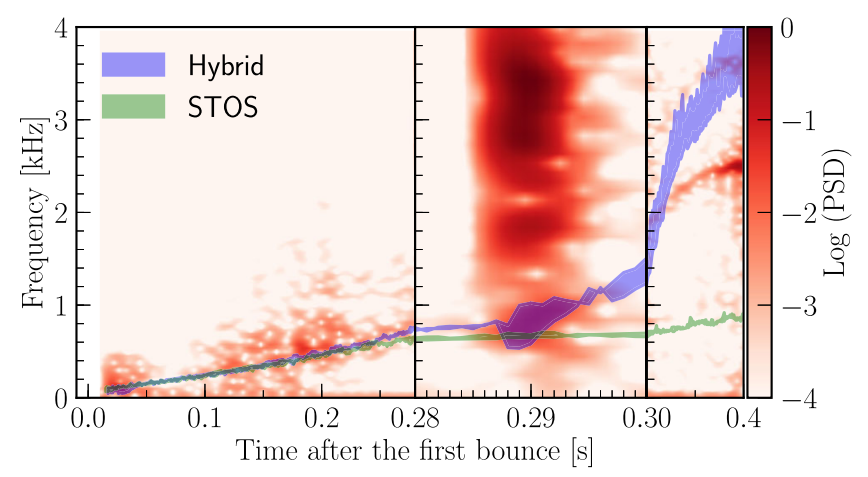

FIG. 3. Color map of the time-dependent power spectral density (PSD) for the GW waveform extracted from the simulation using the hybrid EOS. The color-filled bands track the evolution of the Brunt-Väisälä frequencies at densities between $10^{11}$ and $10^{12} \mathrm{~g} \mathrm{~cm}^{-3}$ for the hybrid (blue) and STOS (green) EOSs.

region from $\sim 10-20 \mathrm{~km}$ to $\sim 5-10 \mathrm{~km}$ (see [30]), which is inside the quasistatic core during the second collapse and bounce. During this time, $f_{\mathrm{BV}}$ peaks at $2900 \mathrm{~Hz}$ near a radius of $10 \mathrm{~km}\left(\rho \simeq 8 \times 10^{13} \mathrm{~g} \mathrm{~cm}^{-3}\right)$ and is closer to the observed GW frequency.

Shortly after $t_{2 b}$, the GW peak frequency drops back to $\sim 1000 \mathrm{~Hz}$ and continues to increase afterwards, albeit at a much faster rate. We find that $f_{\mathrm{BV}}$ has a much larger spread inside the PCS and the GW spectral evolution does not match the track of $f_{\mathrm{BV}}$. After $t_{b}+300 \mathrm{~ms}$, the peak frequency of the dominant $\mathrm{GW}$ emission is closer to $f_{\mathrm{BV}}$ at densities $\sim 5 \times 10^{12} \mathrm{~g} \mathrm{~cm}^{-3}$. Nevertheless, due to the much larger $\rho_{c}$ ( $\gtrsim 4$ times) and compactness of the PCS for the hybrid EOS (Fig. 1), the peak GW frequency is 2-3 times higher than that for the STOS EOS. The GW spectral evolution after $t_{2 b}$ contains information about the structure and evolution of the PCS with a pure quark core, from which one may infer the properties of the quark EOS (e.g., bag constant).

Detection prospect:To estimate the detectability of the GW signals, we calculate the dimensionless characteristic GW strain $\left(h_{\text {char }}\right)$ [37] assuming a distance of $10 \mathrm{kpc}$, and compare it with the sensitivity of Advanced LIGO in Fig. 4. Below $\sim 1000 \mathrm{~Hz}, h_{\text {char }}$ are quantitatively similar for the hybrid and STOS EOSs. At higher frequencies, $h_{\text {char }}$ for the hybrid EOS shows a broad peak between $\sim 2500-4000 \mathrm{~Hz}$, which is also above the detector's sensitivity curve. This part is mainly contributed by the burst associated with the PT-induced collapse, seen from the comparison between the entire $h_{\text {char }}$ and that between $t_{2 b}-3 \mathrm{~ms}$ and $t_{2 b}+7 \mathrm{~ms}$.

We calculate the single-detector signal-to-noise ratio (SNR) of the GW waveforms assuming the optimal orientation using Eq. (1.1) in [37]. If a confident detection requires an SNR of 8, then for the hybrid EOS, inclusion (exclusion) of the burst yields a detection radius of 22 (12) kpc. The detectability of the burst is not significantly better because the current detectors are optimized for GW signals at $\sim 10-1000 \mathrm{~Hz}$. The amplitude of $h_{\text {char }}$ and 


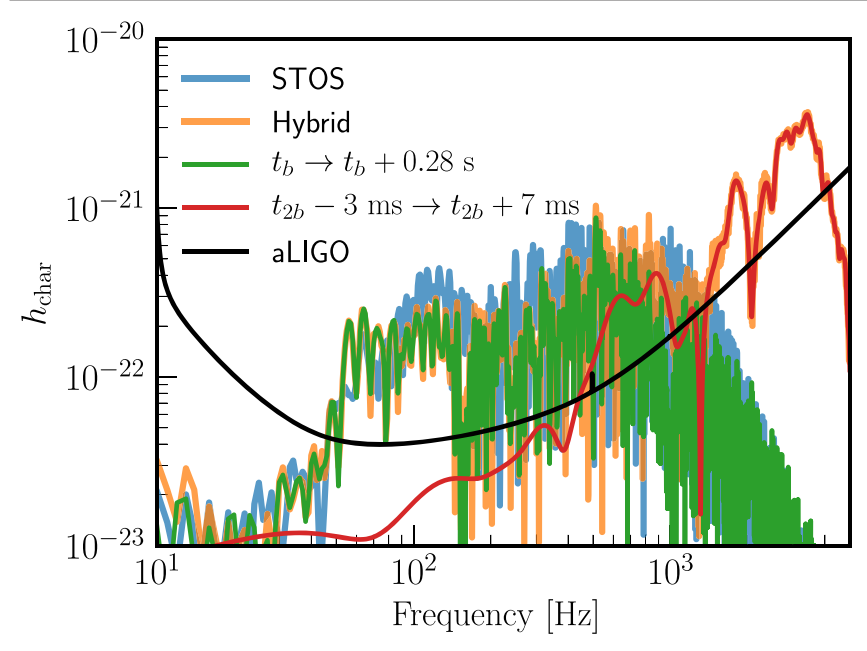

FIG. 4. Dimension-less characteristic strain spectra $\left[h_{\text {char }}(f)\right]$ of the GW signals from $t_{b}$ to $t_{b}+400 \mathrm{~ms}$ with the STOS (blue) and hybrid (orange) EOSs. Also shown are the $h_{\text {char }}(f)$ of the GW signal for the hybrid EOS in the time interval of from $t_{b}$ to $t_{b}+280 \mathrm{~ms}$ (green) and between $t_{2 b}-3 \mathrm{~ms}$ and $t_{2 b}+7 \mathrm{~ms} \mathrm{~ms}$ (red). The black line is the sensitivity spectrum of advanced LIGO [38].

detector noise increase together by a factor of 10 from 100 to $3000 \mathrm{~Hz}$ (Fig. 4). Future-generation detectors, such as the Einstein telescope [39] and the cosmic explorer [40], may consider improving the sensitivity at several $\mathrm{kHz}$ if such signals are targeted (also for $\mathrm{BH}$ forming CCSNe [41]).

In this Letter, the GW waveforms $h_{+}(t)$ are extracted from 2D simulations with the assumption of axisymmetry. In various studies [e.g., $[16,17,42]]$, the amplitude of $h_{+}$in 3D simulations can be 5-10 times smaller than those in their 2D counterparts, which lowers the expectation for the $\mathrm{GW}$ detection. Future study is needed to explore 3D effects for the PT-induced collapse and observables. We expect that the burst associated with the PT-induced collapse would still be present in 3D simulations but with smaller amplitudes.

Discussion.-We present here a specific case in which the PT-induced collapse results in a bounce shock that successfully explodes the mantle. However, for other progenitors [43] or other hybrid EOSs [5], the star may fail to explode and collapse into a black hole (BH) in two scenarios. First, the PCS at the onset of the PT-induced collapse may exceed the maximum mass which the hybrid EOS permits and it directly collapses into a $\mathrm{BH}$. In this case, the GW (and neutrino) burst reported here will be absent. Nevertheless, the existence of free quarks in the PCS might be inferred from the shortening of the $\mathrm{BH}$ formation time [6,44], though it is subjected to the uncertainties of the pure hadronic EOS.

In the second scenario, the second bounce shock is launched but the PCS still collapses into a BH at a later time. In this case, the burst of GWs and neutrinos associated with the PT-induced collapse will be present, followed by the shut off of both signals at $\mathrm{BH}$ formation. This is an interesting possibility to be explored. Moreover, in both cases of $\mathrm{BH}$ formation, if the iron core is rapidly rotating, the inclusion of $\mathrm{PT}$ can produce different $\mathrm{BH}$ ringdown signals compared to those for a hadronic EOS due to the different free fall time of the PCS, which is found in binary NS-merger simulations [8].

Conclusions.-In this Letter, we demonstrate the effects of a first-order QCD PT on the GW signals from a nonrotating CCSN. We find that the PT results in the collapse of the PCS at $\rho_{c} \sim 3.5 \rho_{\text {sat }}$, and the core radiates a loud GW burst in $\lesssim 5 \mathrm{~ms}$. The amplitude of this burst reaches $h_{+}=10^{-20}$ assuming a source distance of $10 \mathrm{kpc}$ and is larger by a factor of $\sim 30$ than other episodes of GW emission (and generally those using a hadronic EOS). The spectrum of this burst shows a broad peak at $\sim 2500-4000 \mathrm{~Hz}$, which is higher than that generally found for CCSNe without the PT-induced collapse. The peak GW frequency following this burst is also much higher $(>1 \mathrm{kHz})$ than that for the hadronic EOS due to the large compactness of the PCS with a pure quark core. Therefore, the PT inside a CCSN can be inferred from the GW detection. However, the louder burst is not necessarily easier to detect because of the increasing noise level of high frequencies for current ground based GW detectors. Nevertheless, the loud, high-frequency burst of GW radiation over a short period of time may be a prime target for future searches of coherent wave burst signals [45].

The hybrid EOS transitions from the hadronic phase to the mixed phase at a low density $\left(\sim \rho_{\text {sat }}\right)$. Reference [7] simulated CCSNe in spherical symmetry using a more physical and complex hybrid EOS (DD2F-SF, transition density $\sim 2.4 \rho_{\text {sat }}$, and the dynamics of the second collapse are similar to our results. Therefore, we expect that the properties of the $\mathrm{GW}$ burst (i.e., the frequency and amplitude) associated with the PT-induced collapse should still be present with a more physical EOS, such as DD2FSF [7,9] or a chiral mean field model [8], which are consistent with the maximum NS mass measurement [46]. A natural extension is to employ such EOSs in multidimensional CCSN simulations. Moreover, progenitor dependence, such as the initial mass and rotation, should be studied to acquire a more comprehensive picture for the effects of a first-order QCD PT on the GWs from CCSNe. Particularly, we expect that if the iron core is rapidly rotating before collapse, the GW burst associated with the PT-induced collapse will be much louder and it may allow the detection for sources farther away.

We thank I. Sagert for making the hybrid equation of state publicly available at the CompOSE website [22]. This work is supported by the Swedish Research Council (Project No. 2018-04575) and by the Research Grant Council of Hong Kong (Project No. 14300317). The simulations were enabled by resources provided by the 
Swedish National Infrastructure for Computing (SNIC) at PDC and NSC partially funded by the Swedish Research Council through grant agreement No. 2016-07213. We also acknowledge the support of the CUHK Central High Performance Computing Cluster, on which parts of the simulations were performed. S. M. C. is supported by the U.S. Department of Energy, Office of Science, Office of Nuclear Physics, under Award No. DE-SC0015904 and No. DE-SC0017955.

*shuai.zha@astro.su.se

[1] E. Witten, Phys. Rev. D 30, 272 (1984).

[2] E. Farhi and R. L. Jaffe, Phys. Rev. D 30, 2379 (1984).

[3] P. Haensel, J. L. Zdunik, and R. Schaefer, Astron. Astrophys. 160, 121 (1986), https://ui.adsabs.harvard.edu/ abs/1986A\%26A...160..121H/abstract.

[4] M. G. Alford, S. Han, and M. Prakash, Phys. Rev. D 88, 083013 (2013).

[5] I. Sagert, T. Fischer, M. Hempel, G. Pagliara, J. Schaffner-Bielich, A. Mezzacappa, F. K. Thielemann, and M. Liebendörfer, Phys. Rev. Lett. 102, 081101 (2009).

[6] K. Nakazato, K. Sumiyoshi, and S. Yamada, Astron. Astrophys. 558, A50 (2013).

[7] T. Fischer, N.-U. F. Bastian, M.-R. Wu, P. Baklanov, E. Sorokina, S. Blinnikov, S. Typel, T. Klähn, and D. B. Blaschke, Nat. Astron. 2, 980 (2018).

[8] E. R. Most, L. J. Papenfort, V. Dexheimer, M. Hanauske, S. Schramm, H. Stöcker, and L. Rezzolla, Phys. Rev. Lett. 122, 061101 (2019).

[9] A. Bauswein, N.-U.F. Bastian, D. B. Blaschke, K. Chatziioannou, J. A. Clark, T. Fischer, and M. Oertel, Phys. Rev. Lett. 122, 061102 (2019).

[10] T. Fischer, M.-R. Wu, B. Wehmeyer, N.-U. F. Bastian, G. Martínez-Pinedo, and F.-K. Thielemann, Astrophys. J. 894, 9 (2020).

[11] B. P. Abbott, R. Abbott, T. D. Abbott, M. R. Abernathy, F. Acernese, K. Ackley, C. Adams, T. Adams, P. Addesso, R. X. Adhikari et al., Phys. Rev. D 94, 102001 (2016).

[12] C. D. Ott, Classical Quantum Gravity 26, 204015 (2009).

[13] H. Dimmelmeier, C. D. Ott, H. T. Janka, A. Marek, and E. Müller, Phys. Rev. Lett. 98, 251101 (2007).

[14] C. L. Fryer and K. C. B. New, Living Rev. Relativity 14, 1 (2011).

[15] K. Hayama, T. Kuroda, K. Nakamura, and S. Yamada, Phys. Rev. Lett. 116, 151102 (2016).

[16] E. P. O'Connor and S. M. Couch, Astrophys. J. 865, 81 (2018).

[17] D. Radice, V. Morozova, A. Burrows, D. Vartanyan, and H. Nagakura, Astrophys. J. Lett. 876, L9 (2019).

[18] L. M. Lin, K. S. Cheng, M. C. Chu, and W. M. Suen, Astrophys. J. 639, 382 (2006).

[19] E. B. Abdikamalov, H. Dimmelmeier, L. Rezzolla, and J. C. Miller, Mon. Not. R. Astron. Soc. 392, 52 (2009).

[20] H. Dimmelmeier, M. Bejger, P. Haensel, and J. L. Zdunik, Mon. Not. R. Astron. Soc. 396, 2269 (2009).

[21] I. Sagert, T. Fischer, M. Hempel, G. Pagliara, J. Schaffner-Bielich, F. K. Thielemann, and M. Liebendörfer, J. Phys. G 37, 094064 (2010).
[22] https://compose.obspm.fr/eos/75/.

[23] H. Shen, H. Toki, K. Oyamatsu, and K. Sumiyoshi, Prog. Theor. Phys. 100, 1013 (1998).

[24] N. K. Glendenning, Phys. Rev. D 46, 1274 (1992).

[25] M. Hempel, O. Heinimann, A. Yudin, I. Iosilevskiy, M. Liebendörfer, and F.-K. Thielemann, Phys. Rev. D 94, 103001 (2016).

[26] B. Fryxell, K. Olson, P. Ricker, F. X. Timmes, M. Zingale, D. Q. Lamb, P. MacNeice, R. Rosner, J. W. Truran, and H. Tufo, Astrophys. J. Suppl. Ser. 131, 273 (2000).

[27] E. P. O'Connor and S. M. Couch, Astrophys. J. 854, 63 (2018).

[28] S. E. Woosley, A. Heger, and T. A. Weaver, Rev. Mod. Phys. 74, 1015 (2002).

[29] A. Marek, H. Dimmelmeier, H. T. Janka, E. Müller, and R. Buras, Astron. Astrophys. 445, 273 (2006).

[30] See the Supplemental Material at http://link.aps.org/ supplemental/10.1103/PhysRevLett.125.051102 for the general relativistic approximations, test of potential numerical artifacts, resolution dependence, spatial distribution of $h_{+}$, and the GW spectrogram for the STOS EOS, which includes Refs. [31,32].

[31] M. A. Pajkos, S. M. Couch, K.-C. Pan, and E. P. O'Connor, Astrophys. J. 878, 13 (2019).

[32] E. O'Connor and C. D. Ott, Classical Quantum Gravity 27, 114103 (2010).

[33] M. Obergaulinger and M. Á. Aloy, Mon. Not. R. Astron. Soc. 492, 4613 (2020).

[34] L. S. Finn and C. R. Evans, Astrophys. J. 351, 588 (1990).

[35] A. Torres-Forné, P. Cerdá-Durán, M. Obergaulinger, B. Müller, and J. A. Font, Phys. Rev. Lett. 123, 051102 (2019).

[36] B. Müller, H.-T. Janka, and A. Marek, Astrophys. J. 766, 43 (2013).

[37] É. É. Flanagan and S. A. Hughes, Phys. Rev. D 57, 4535 (1998).

[38] L. Barsotti, P. Fritschel, M. Evans, and S. Gras (LIGO Collaboration), Technical Report LIGO-T1800044-v5, 2018, https://dcc.ligo.org/LIGO-T1800044/public.

[39] M. Punturo, M. Abernathy, F. Acernese, B. Allen, N. Andersson, K. Arun, F. Barone, B. Barr, M. Barsuglia, M. Beker et al., Classical Quantum Gravity 27, 194002 (2010).

[40] B. P. Abbott, R. Abbott, T. D. Abbott, M. R. Abernathy, K. Ackley, C. Adams, P. Addesso, R. X. Adhikari, V. B. Adya, C. Affeldt et al., Classical Quantum Gravity 34, 044001 (2017).

[41] K.-C. Pan, M. Liebendörfer, S. M. Couch, and F.-K. Thielemann, Astrophys. J. 857, 13 (2018).

[42] H. Andresen, B. Müller, E. Müller, and H. T. Janka, Mon. Not. R. Astron. Soc. 468, 2032 (2017).

[43] E. O'Connor and C. D. Ott, Astrophys. J. 730, 70 (2011).

[44] A.d.S. Schneider, E. O'Connor, E. Granqvist, A. Betranhandy, and S. M. Couch, Astrophys. J. 894, 4 (2020).

[45] S. Klimenko, I. Yakushin, A. Mercer, and G. Mitselmakher, Classical Quantum Gravity 25, 114029 (2008).

[46] J. Antoniadis, P. C. C. Freire, N. Wex, T. M. Tauris, R. S. Lynch, M. H. van Kerkwijk, M. Kramer, C. Bassa, V. S. Dhillon, T. Driebe et al., Science 340, 1233232 (2013). 